\title{
Study on Turning Arabic Text into Spoken Words
}

\author{
Abdulwahhab F. Shareef \\ abdalsied2017@gmail.com
}

Riyadh Z. Mahmoud

riyadh_1970@yahoo.com

Department of Computer Science

College of Computer Science and Mathematics

University of Mosul, Mosul, Iraq

Received on: $23 / 02 / 2021$

Accepted on: 04/04/2021

\begin{abstract}
Language was a means of communication between members of a single community, so that some of them would express to each other their ideas and thoughts, and a common characteristic of that society was defined, and the origin of the word was derived from (rhetoric, idle), if he repealed the matter and spoke about it, and the ancients knew it that language: (It is what expresses It contains all the people for their belongings). The ancients went in their definition of a language to the characteristics of their language with which they communicate, without referring to the Arabic language as a language among the living languages that are circulating among them. Arabs and Arabs (whoever inhabited the country and its island, or uttered the language of its people), and to it returns the percentage of the Arabic language, which is one of the Semitic languages. Which spread in the Arabian Peninsula, and the writing came to denote what is in the minds of notables.

This research presents a computer application that depends on human input to pronounce the Arabic letters

The system consists of two phases, the first stage is the axis of creating a database for Arabic language characters and their storage locations, as well as the type of formulas for those letters when the initial processing was performed.

The second stage is the process of comparing the entered letter from the text with the corresponding sound and placing it in a storage so that we can then process it. In the practical part of the research, we used a comparison between the results of four methods to obtain the least possible execution time with the least pauses in speech, which are the combinative method, the smoothing method, the method of nesting speech, and a hybrid method between smoothing and interfering together.

We start by entering in the input text box and using the SpellLetter function, which we use for the purpose of processing and pronunciation, where the input is of three types, either it is a char, or it is an array of characters (String), or it is numbers between zero and nine Num), and in the case that the entry was not For these three types, the entry is wrong, for example, non-Arabic letters or special symbols, for example.,And if the entry was correct, each letter is taken with its accent. This means that we take two positions each time.

Using Matlab (R 2018a) to build the proposed system and it was implemented using a computer. Portable running under the environment of the operating system (Microsoft Windows 10).
\end{abstract}

Keywords: Sound Recording, Analysis, Synthesis, Overlap, Smoothing Speech. 


\section{دراسة حول تحويل النص العربي الى كلمات منطوقة}

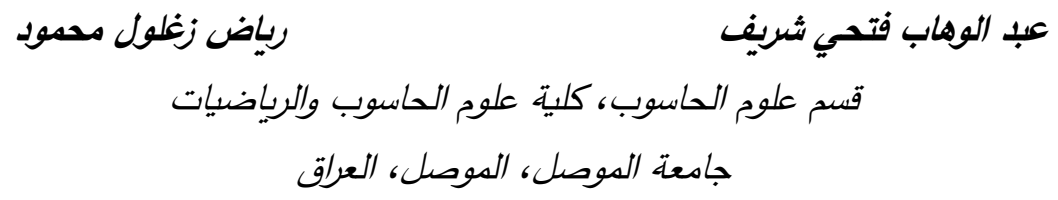

\section{الملخص}

اللغة وسيلة للتواصل بين أفراد المجتمع الواحد، فيعبِّر بعضهم لبعض عن أفكارهم وخواطرهم، وتحدد صفة

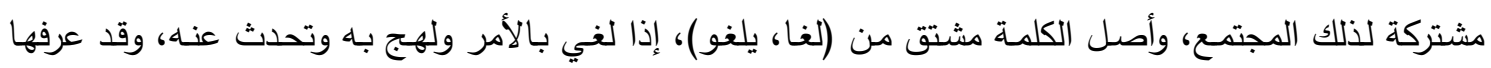

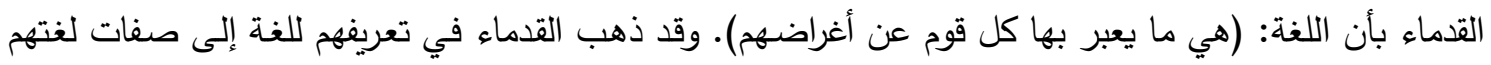
التي يتواصلون بها، دونما إثارة إلى اللغة العربية كلغة بين اللغات الحية المتداولة بينهم، فالعَرَب والعُرب (كل من لهن

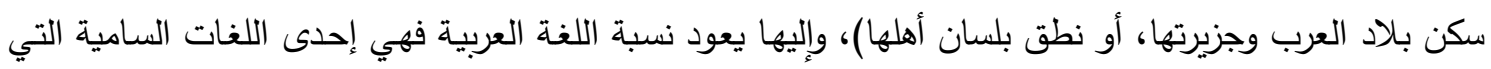

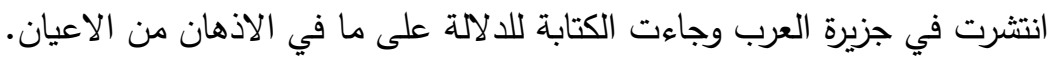

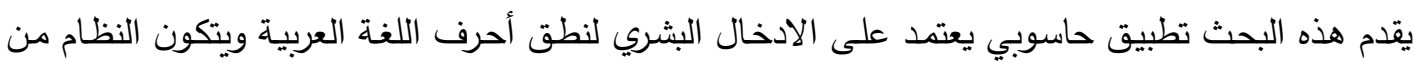

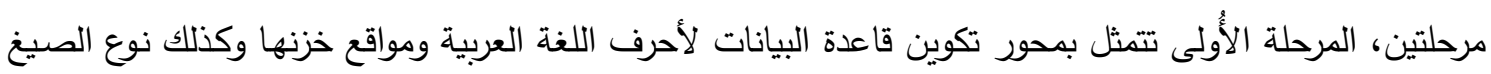
لتلك الحروف إذ تم إجراء المعالجات الأولية. اما المرحلة الثانية تتمثل بعملية مقارنة الحرف المدخل من النص مع الصوت المقابل لـه ووضعها في مخزن

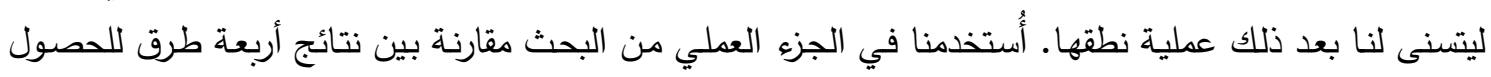
على اقل وقت تتفيذ مدكن مع اقل توقفات عند نطق وهي الطريقة التجميعية وطريقة التتعيم وطريقة التداخل الكلام وطريقة هجينة بين التتعيم والتذاخل معا. نبدأ بالإدخال في مربع النص المدخل وعن طريق دالة SpellLetter والتي نستخدمها لغرض المعالجة والنطق، حيث يكون الادخال على ثلاثة انواع اما يكون حرف (char) او يكون مصفوفة حروف (String) او يكون ارقام بين

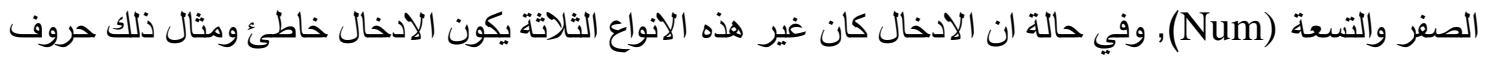

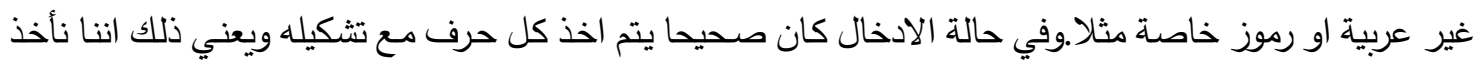
موقعين اثثين في كل مرة. باستخدام لغة الماتلاب (R 2018a) لبناء النظام المقترح وتم تتفيذه باستخدام حاسوب محمول يعمل تحت

بيئة نظام التشغيل (Microsoft Windows 10). الكلمات المفتاحية: تسجيل الصوت، تحليل الكلمات المدخلة، التجميع، التداخل، التتعيم، النطق.

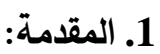

تعد اللغة العربية من اللغات القديمة الثائعة في العديد من بقاع الأرض، فهي لغة القران الكريم ويتحدث بها

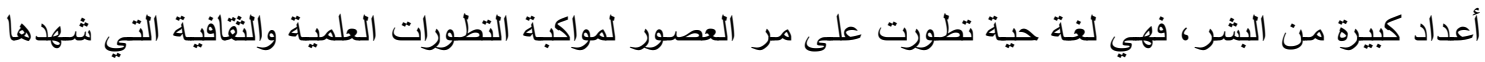


العالم؛ إذ كانت في العصور الوسطى لغة العلوم والفنون ويدرسها طلبة العلم في مختلف منتديات العلم والمعرفة في

الدول العربية في المشرق والمغرب حين كانت قبلة الطلبة والأساتذة في مختلف المجالات العلمية والأدبية (1).

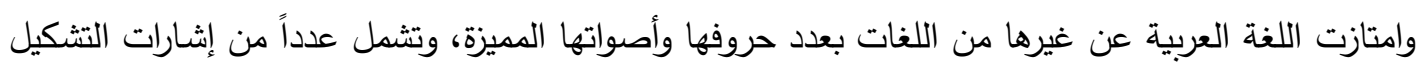

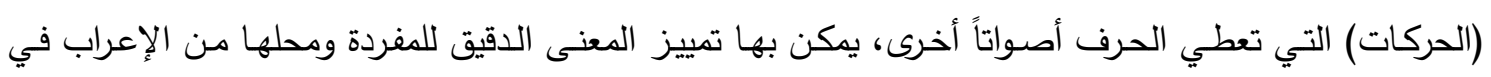

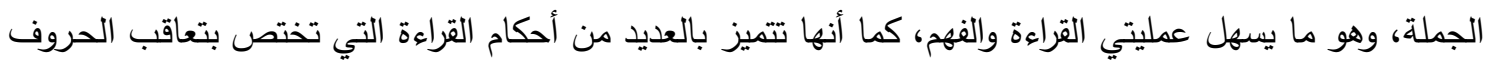
العربية في ظل قواعد وضعها العلماء للوصول إلى النطق الصحيح للكلمة، قياساً على ما جاء في القرآن الكريم

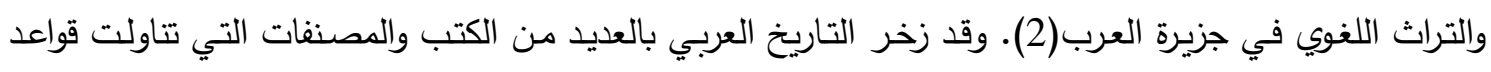

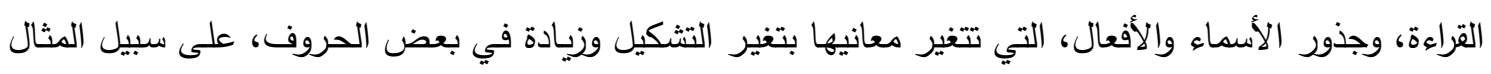
لا الحصـر كتـاب الخصـائص لأبـن جني (ت: 392هـ) في النحو (3)، وكتاب مشتبهات القـرآن للكسـئي(ت:

189هـ) في التجويد والقراءات(4). وهو أمر من الصعوبة بمكان يتطلب المزيد من الدراسة والتعلم واستخدام الوسائل المتاحة لتسهيل قواعد القراءة

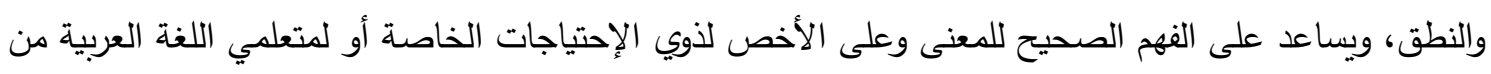

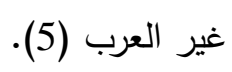

وبتطور تقنيات الحوسبة وقواعد البيانات والملفات متعددة الوسائط، صـار من السهل أن تعد البرامج الخدمية

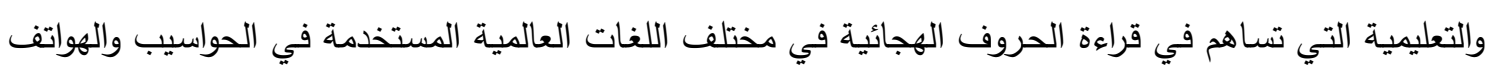
النقالة وغيرها من أنواع الأجهزة الإلكترونية، وساهمت في ارشاد المتعلم لهذه اللغات إلى النطق السليم للكلمات التي

تشكلها (6).

ولقد كانت اللغة العربية من اللغات المستخدمة في تقنيات الحاسوب، وتعددت الدراسات التي حاولت محاكاة

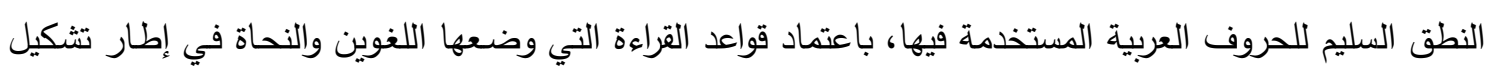

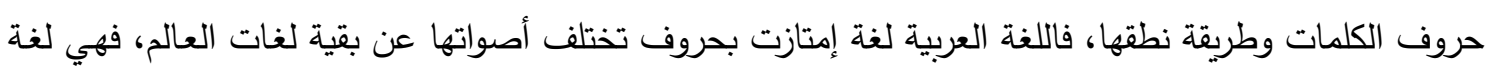
الضاد التي لا ينطق بها سوى العرب، وهي اللغة التي تشمل العديد من قواعد القراءة، مثل تمييز الحرف المشكل

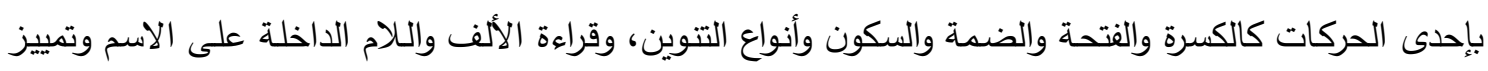

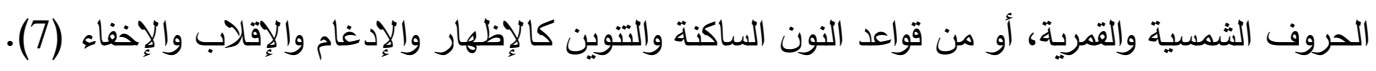

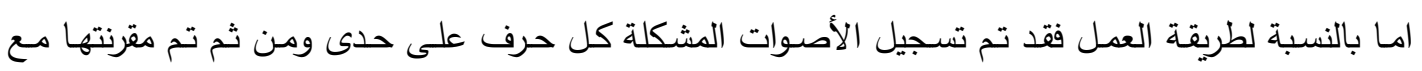
الحروف المدخلة من النص المطلوب نطقه بعد تقسيم الكلمات الى حروف منفصلة ومن ثم وضعها في مخزن

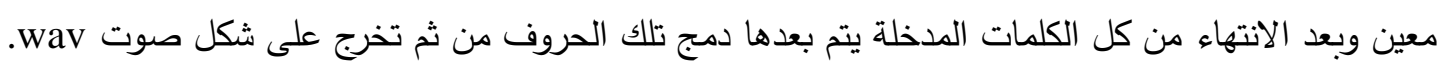

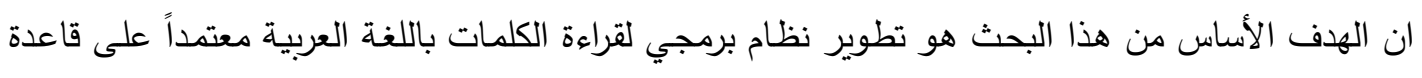
بيانات مرتبطة بقواعد بيانات للملفات الصوتية التي تمثل النطق بالأحرف حسب القاعدة اللغويـة للنطق. وفي سبيل

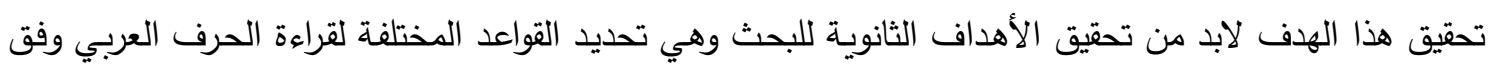

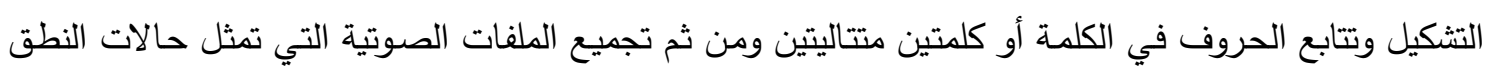
بالحروف في قواعد بيانات منتظمة، لاستخدامها في عملية النطق حيث يتم استخدام نظم تشفير الحروف العربية وحركات التثكيل الخاصة بها في التعرف على الحرف وبعدها يتم تصميم الدوال الخاصة بالتعرف على الحالة التي يُنطق بها الحرف أو الحروف المتتابعة في إطار الكلمة أو الكلمتين حيث يتم تصميم واجهة برمجية ملائمة لإدخال النص العربي أو الكلمة العربية لاستخدام الدوال البرمجية وربطها بقواعد البيانات الخاصة بالصوت، يتم إنم النطق بها. 
من خلال العودة إلى أدبيات حول موضوع الدراسة نجد أن هناك العديد من الدراسات التي تتاولت موضوع النطق المحوسب للأحرف الهجائية في اللغات المختلفة، وهي كما يأتي: استخدم الباحث (9) طريقة بديلة في إعداد الوسائط لتدريس الإستماع، والتي يمكن أن تستوعب فئي ما تقتضيه

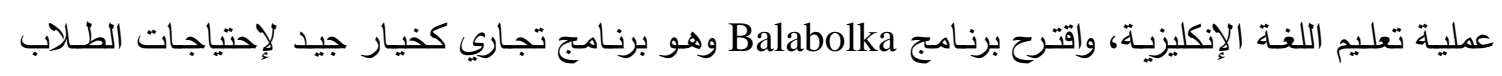

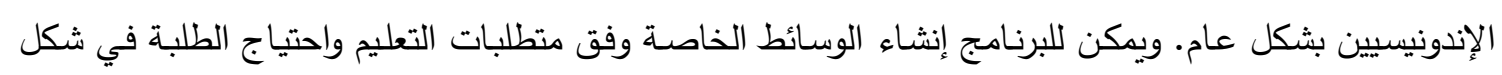
ملفات صوتية ذات الإمتدادات MP4, OGG, WMA WAV, MP3. ويسهم البرنامج بشكل فعال في تتمية

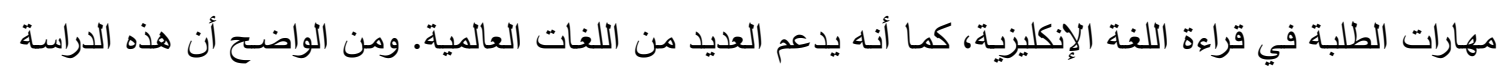
عنيت بدراسة اللغة الإنكليزية التي لا تتمتع بذات الخصائص التي تتمتع بها اللغة العربية.

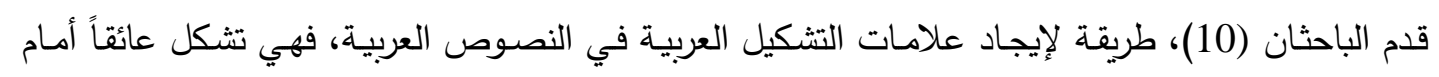

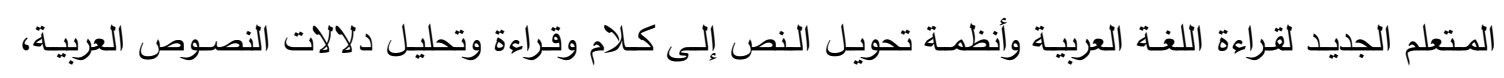

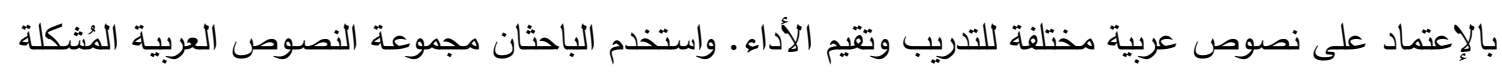

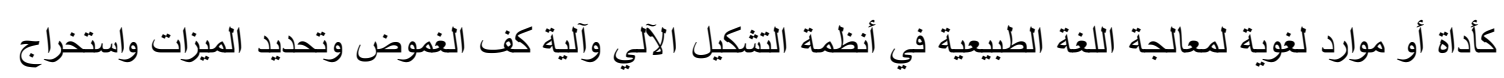

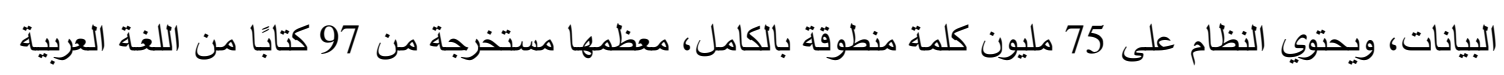

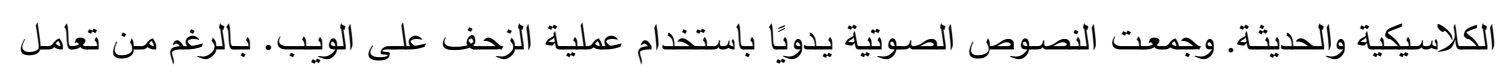

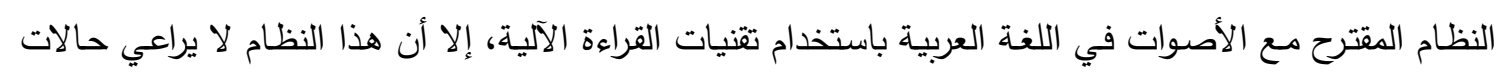

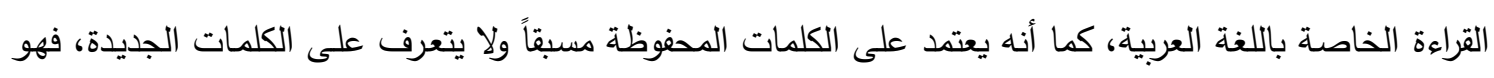
لا يعتمد على تميز الأحرف في النص العربي، ولا يولد الأصوات حسب الحالة في القراءة.

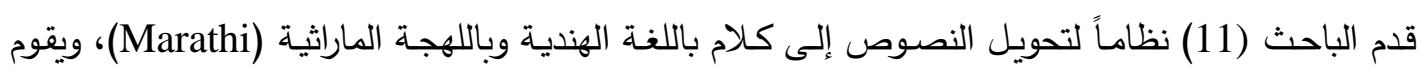
البرنامج باختيار وحدة الكلام (الكلمة) بالأسلوب المتسلسل ويحولها إلى صوت باعتماد التسجيلات الصوتية المسبقة

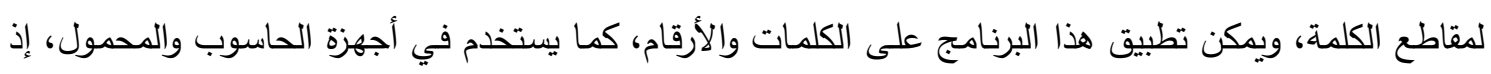

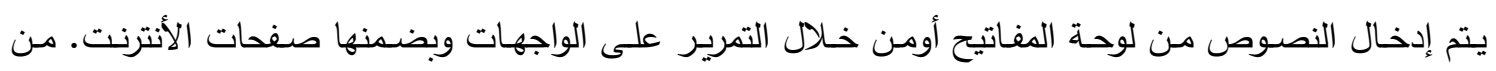
الواضح أن اللغة المستخدمة في هذا البرنامج هي ليست اللغة العربية، ولكن أسلوب الاختيار المتسلسل هو نقطة التهن

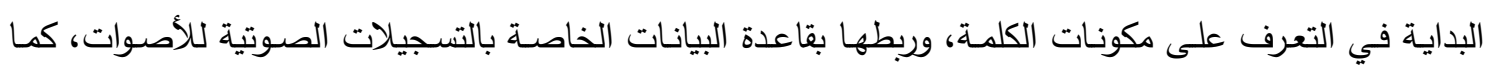
يشير البحث إلى إمكانية تطبيق تقنية تحويل الكلام إلى نص في اللغات الأخرى وحسب الحاجة فئل في تلك اعلك اللغات. قدم الباحثان (12) طريقة حديثة في تحويل النص إلى كلام باستخدام طريقة من تقنيات الثبكة العصبية

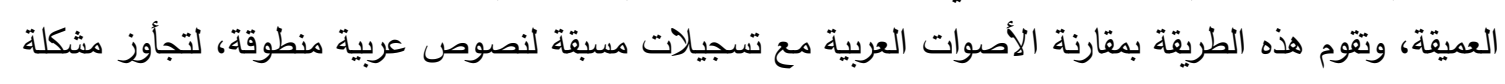

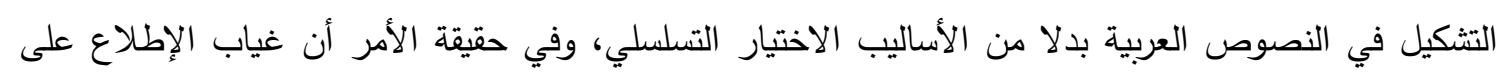

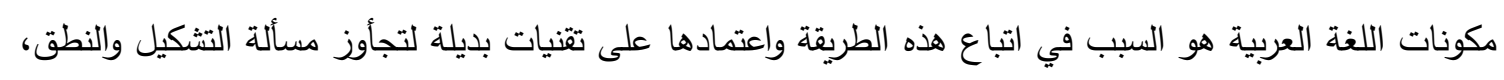

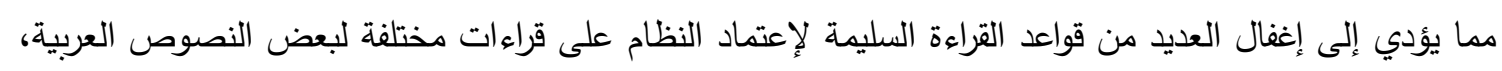
التي قد يعنى القارئ بقواعد اللغة أو ليس لديه إطلاع عليها من الأساس. قذّم الباحثون (13) طريقة جديدة لتمييز مدة أصوات الأحرف العربية في إطار نمذجة اللغة العربية، لتسهيل التباتيل

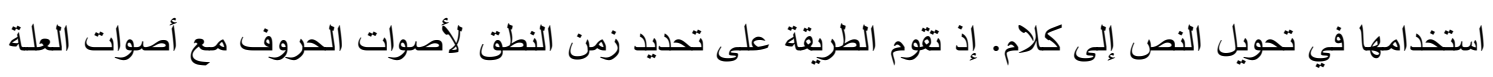


الطويلـة في حروف العلة، وأصسوات العلة القصيرة مـع الحركات، وأصسوات الحروف السـاكن. واستخدم الباحثون

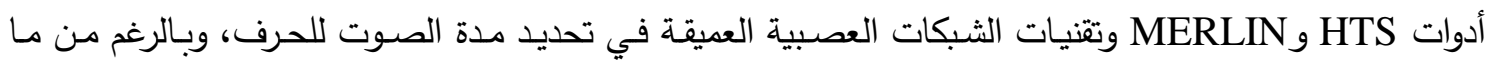
توصلت إليه الطريقة من نمذجة أفضل، الا انها تعاني من القصور في حركات التشكيل مثل التتوين بأنواعه، كما

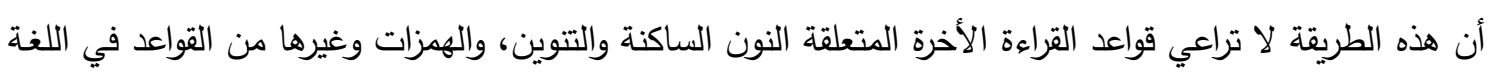
العربية. ويعزى سبب ذلك إلى ضيق المعرفة بقواعد القراءة في اللغة العربية. وقدم الباحث (14) نظاماً لتحويل الكلام إلى نص في اللغة العربية، وقد اجتهد باعتماد قواعد القراءة والتجويد

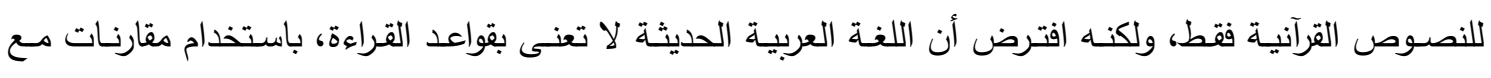

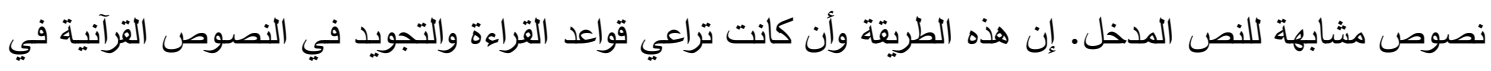
تحويل الكلام إلى نص، فإنها لأ تراعي الكلام الاعتيادي وبلهجاته المختلفة. إذ إن المتفحص للكلام باللغة لتحة العربية

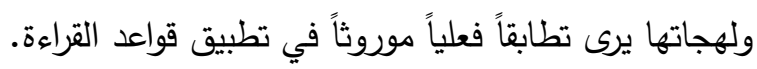

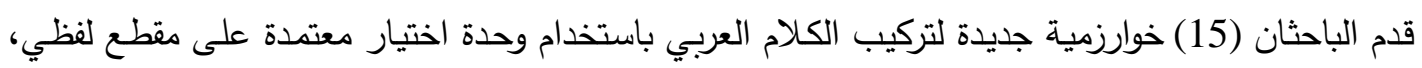

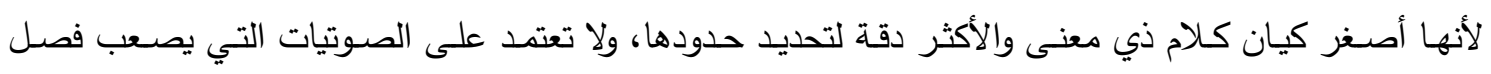

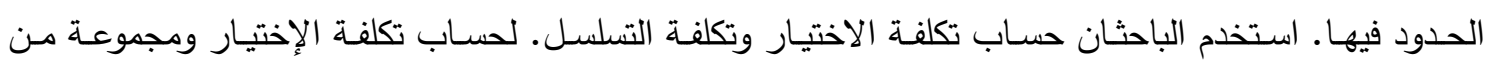
الأصوات واللغوية، مستخدمين متوسط فرق المعلمات العامة بين الوحدات المجأورة. واحتفظا بالتسلسل الأقل تكلفة

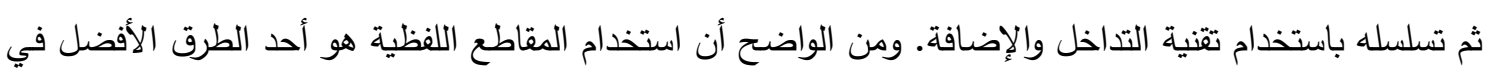

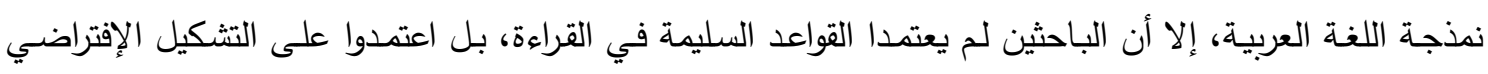
للكلمة، ولم يأتِ تقييم الأداء مواكباً لقواعد القراءة، بل اعتمد حساب الكلفة في تقييم عملية تحويل النص إلى كلام.

3. ادخال النص العربي وانواعه المقبولة:

في بداية الامر نبدأ بالإدخال في مربع النص المدخل وعن طريق دالة SpellLetter والتي نستخدمها لغرض المعالجة والنطق، حيث يكون الادخال على ثلاثة انواع اما يكون حرف (char) او يكون مصفوفة حروف (String) او يكون ارقام بين الصفر والتسعة(Num ), وفي حالة ان الادخال كان غير هذه الانواع الثلاثة يكون الادخال خاطئ ومثال ذلك حروف غير عربية او رموز خاصة مثلا. وفي حالة الادخال كان صحيحا يتم اخذ كل حرف مع تثكيله ويعني ذلك اننا نأخذ موقعين اثثين في كل مرة. يتم معرفة الحرف الموقع المؤشر عليه هل هو حرف ام حركة وذلك عن طريق ال Ascii code حيث يتم استخدام مفاتيح الكيبورد لأظهار الحركات فمثلا. Shift+Q لأظهار الفتحة نستخدم لإطورد لأظهار الضمة نستخدم لأطهار لأظهار الكسرة نستخدم Shift+A shift+X لأظهار السكون نستخدم لأخدم نستخ لأظهار تتوين الفتح نستخدم shift+W shift+R لأظهار تتوين الضم نستخدم تلوين نلخدم

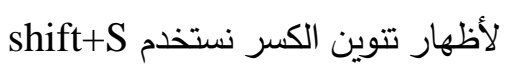


هذا في حالة ان كل الحروف محركة اما إذا كان احد الحروف غير محرك فعند ذلك نقوم بإضـافة سكون لذلك الحرف ثم اجراء باقي العمليات عليه وبعد ذلك نقوم بأخذ حرف مع تثكيله ثم نقارنه مع ملف الاصوات المخزونة ثم فئ

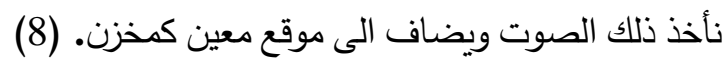
4. شرح مبسط لمكونات مخطط تحويل النص الى كلمات: تسجيل الصوت (Sound Recording): وهو عملية ادخال الصوت عن طريق التسجيل او سحب المقاط

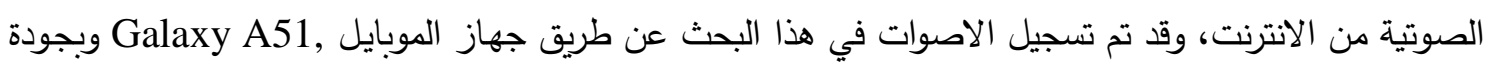
تسجيل عالية تبلغ تحلغ 256 Kbps. تحليل الصوت (Analysis): يتم تحليل الصوت المدخل وتقسيمه الى اجزاء كل جزء يحتوي على حرف وحركة. قاعدة الصوتيات المخزنة (Database): بعد اكمال كافة الاجزاء الخاصة بالأحرف مع حركاتها يتم خزها في مخزن معين لغرض استدعاء الحرف المحرك لاحقا. نماذج الاصوات (Phonetic Symbols): نماذج الكتابة المدخلة. المواصفات المستهدفة (Target Specification): عملية تقطيع الكلام الى أحرف مشكَّة. اختيار الوحدات الصوتية (Unit Selection): اخذ كل صوت مقابل كل حرف في الجملة. التجميع او التركيب (Synthesis): تجميع الاصوات في مخزن معين لكي يتم نطقها فيما بعد. الصوت (Speech): وهو الكلام المنطوق الناتج من عملية تجميع الاحرف والذي يكون قريب من النطق البشري. 5. تصميم النظام المقترح وتنفيذه

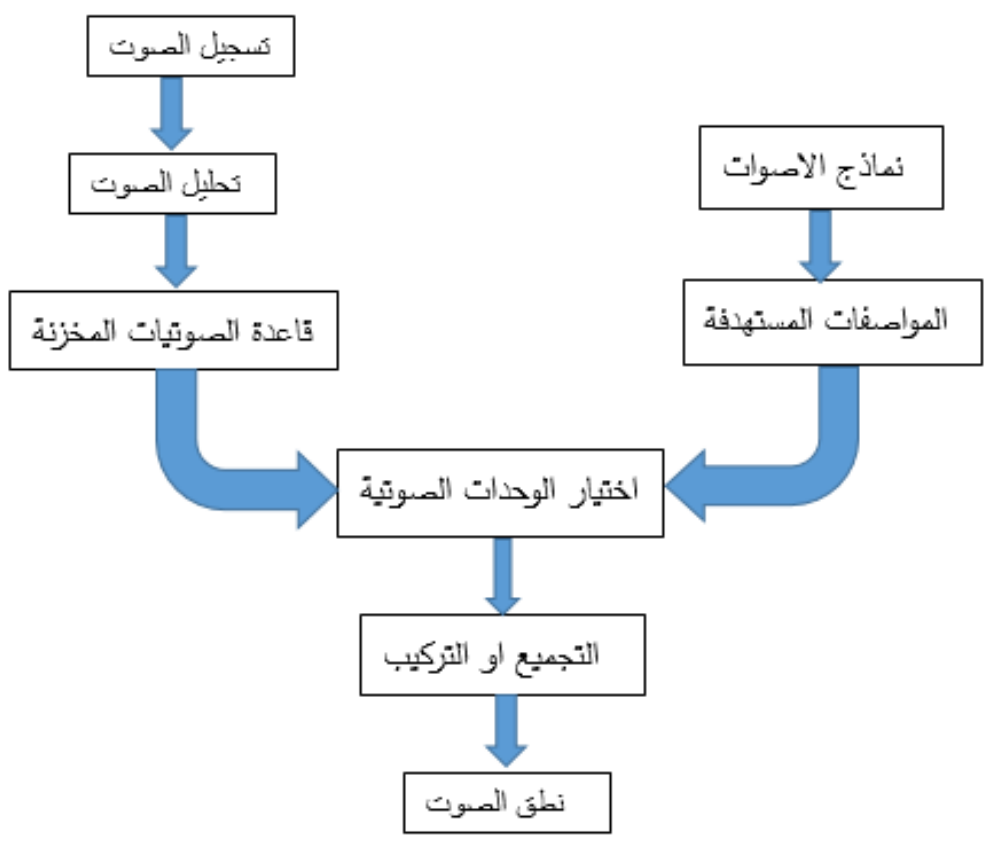

مخطط (1) تحويل النص المطبوع الى كلمات

6. شرح الواجهة للبرنامج المقترح: والواجهة الثانية تحتوي على سبعة عناصر وهي 
• النص المدخل: وهو النص العربي الذي نقوم بكتابته داخل مربع النص قبل اجراء اي عملية معالجة له. • • النص المعالج: وهو الاخراج الناتج بعد اجراء عمليات حاسوبية على النص المدخل. • المعالجة والنطق: وهو اهم زر في هذه الواجهة حيث يستلم من مربع نص الادخال وبعد اجراء عدد من العمليات الحاسوبية على النص يظهر النص المعالج في مربع نص المعالج الادخال . • زر الايقاف: الغاية من هذا الزر هو ايقاف المعالجة او النطق باي وقت اثناء التنفيذ.

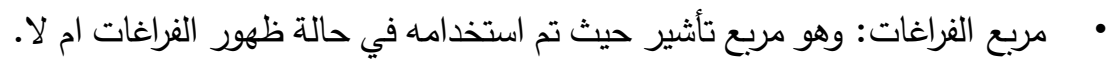

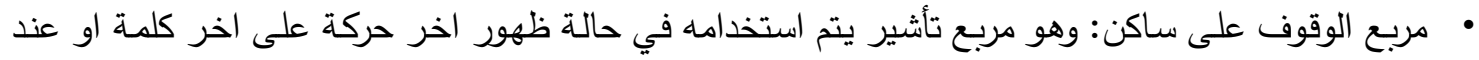

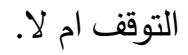

• مربع التداخل: وهو مربع تأثير يتم استخدامه في حالة اجراء عملية التداخل، حيث تم استخدام عدد 2000 وحدة صوتية في بحثنا هذا. • مربع التتعيم: وهو مربع تأثير يتم استخدامه في حالة اجراء عملية التتعييم على الاشارة الصوتية ككل. • مربع تمثيل الاشارة: وهو مربع تمثيل البيانات الصوتية على شكل اشارة. • عدد الاصوات الصامتة: وهو رقم لعدد الاصوات الصامتة على طول الموجة الصوتية. وكما موضح في الثكل (1-3) لواجهة الادخال والمعالجة.

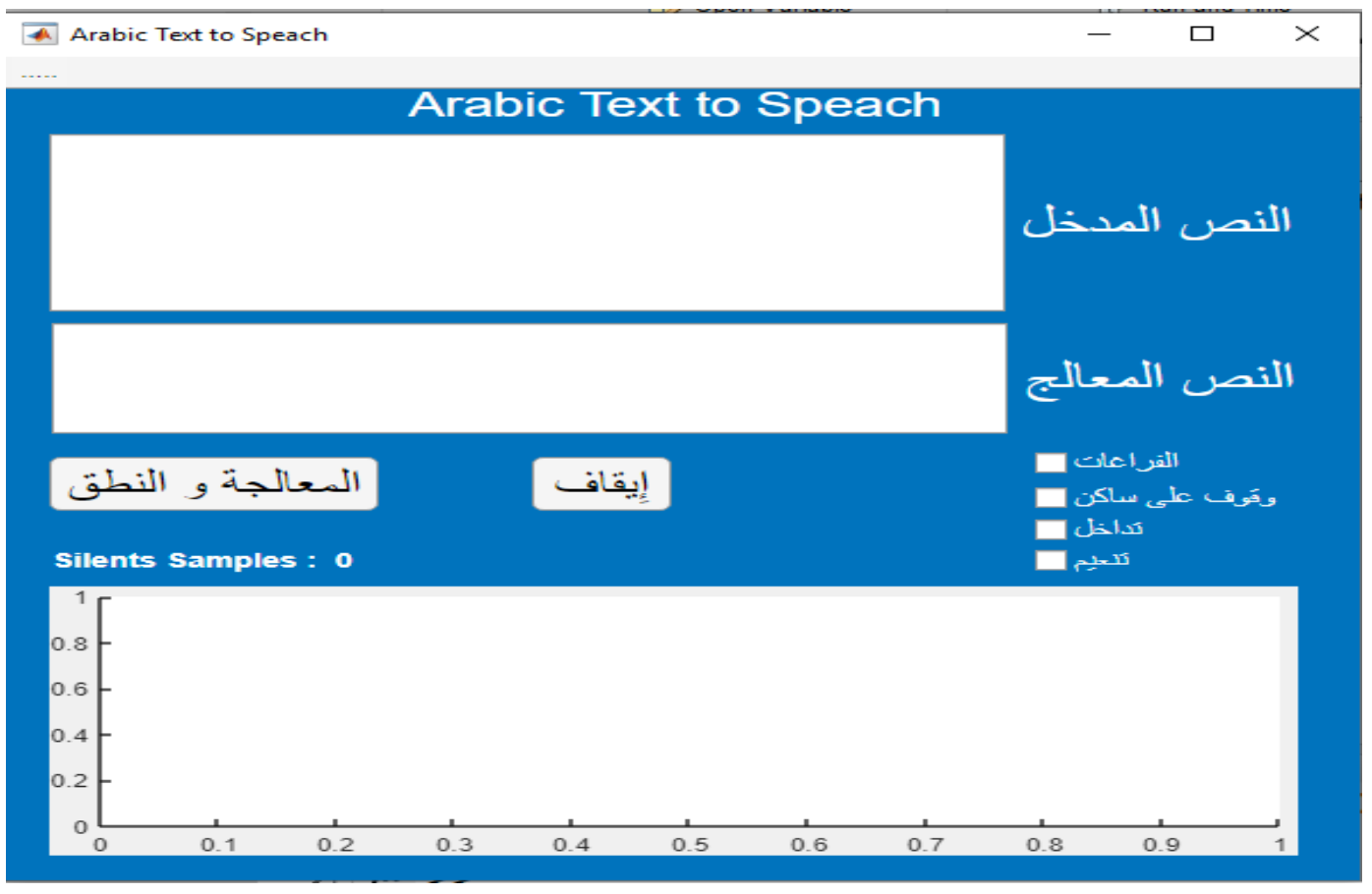

الثكل (2) واجهة الادخال والمعالجة. 
2.8 تطبيق مثال باستخدام البرنامج المقترح:

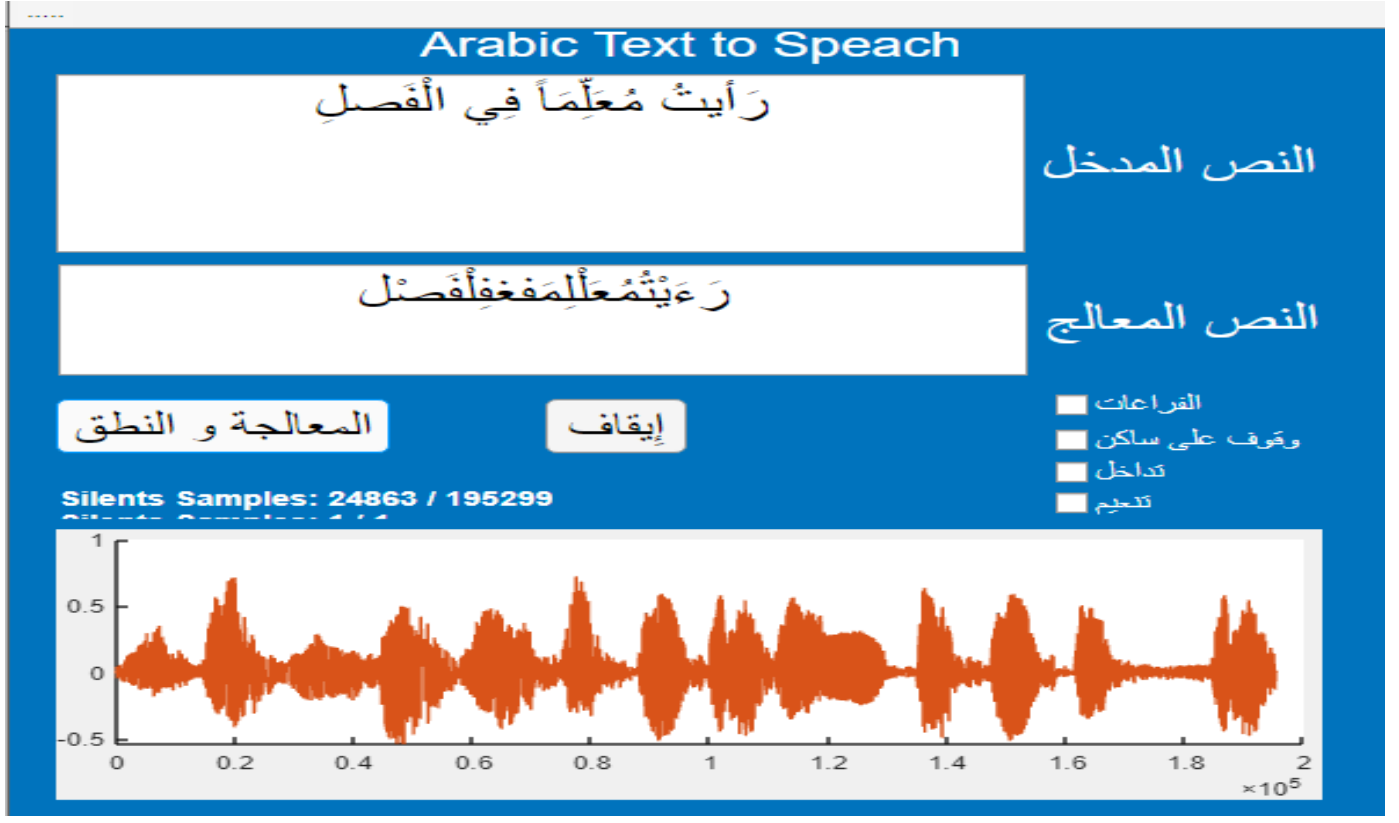

الثكل (3) تطبيق مثال باستخدام البرنامج المقترح

$$
\begin{aligned}
& \text { في هذا الجملة تم تطبيق } 11 \text { قانون وهي: }
\end{aligned}
$$

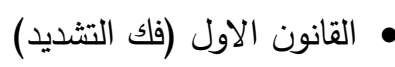

$$
\begin{aligned}
& \text { • القانون الثاني (حذف الالف من ال التعريف) } \\
& \text { • القانون السادس (التتوين) }
\end{aligned}
$$

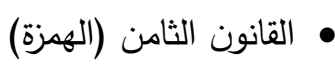

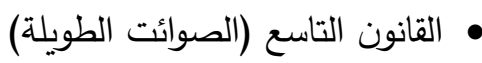

$$
\begin{aligned}
& \text { • (القانون الحادي عشر (النون) }
\end{aligned}
$$

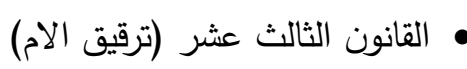

$$
\begin{aligned}
& \text { • • القانون الرابع عشر (تفخيم الراء) } \\
& \text { • القانون السابع عشر (تقصير الصوائت القصيرة) } \\
& \text { • القانون الثامن عشر (تحويل الصوائت الطويلة الى قصيرة) } \\
& \text { • القانون التاسع عشر (حذف اخر صائت في العبارة) }
\end{aligned}
$$

7. - 1 بع الطرق لحل مشكلة التقطيع

بعد تطبيق البرنامج المقترح لنطق الكلام ظهرت لدينا مشكلة وهي ظهور بعض التقطيعات الصوتية بعد عملية الدمج ولكي يتم حل هذه المشكلة كليا او التقليل من تلك التقطيعات الصوتية تم استخدام ثلاث طرق إضـافية وهي التداخل والتتعيم وطريقة ثالثة مقترحة وهي الدمج بين التذاخل والتتعيم بالإضافة الطريقة التجميعية. 1- طريقة التجميع: وهي عملية تجميع الاحرف المنطوقة في مخزن معين لغرض تجميعها ونطقها دفعة واحدة. 
2- طريقة التداخل: وهي عملية تداخل بين نهاية الإثارة الصوتية للحرف السابق مع بداية الحرف الاحق بنسبة

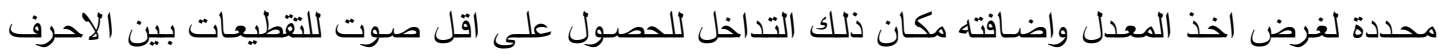

3- طريقة التتعيم: وهي تعديل نقاط البيانات للإشارة الصوتية بحيث يتم تقليل النقاط الفردية التي تكون أعلى من النقاط المجاورة مباشرة (من المفترض بسبب الضوضـاء) ، وزيادة النقاط الأقل من النقاط المجاورة. يؤدي هذا بطبيعة الحال إلى إثـارة أكثر سلاسـة (واستجابة خطوة أبطأ للإشـارة للتغييرات). طالما أن الإثـارة الأساسية الحقيقية سلسة فعليًا، فلن يتم تثويه الإثارة الحقيقية كثيرًا عن طريق التتعيم، ولكن سيتم تقليل ضوضـاء التردد

العالي. (16)

4- طريقة دمج بين التداخل والتتعيم: يتم الدمج بين الطريقتين السابقتين للحصول على اقل صوت للتقطيعات بين الاحرف المنطوقة.

سنأخذ كلمة " أحمَد " كمثال تطبيقي صوتي ونطبق الطرق الاربعة عليها ينتج لنا:
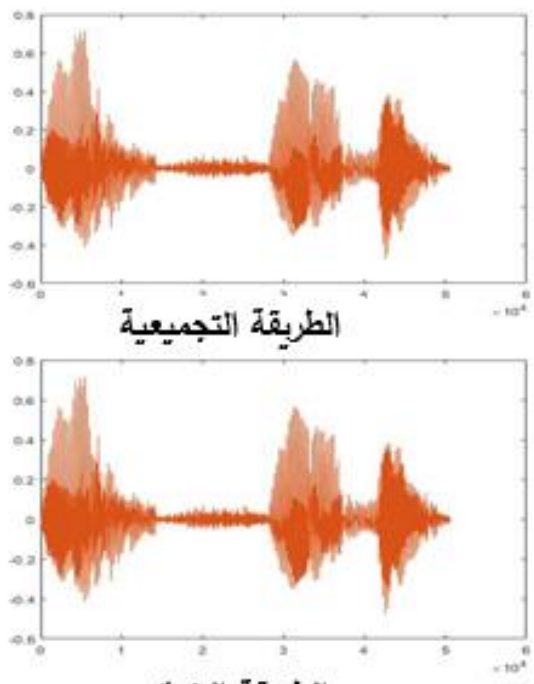

الطربقة التنئيّم

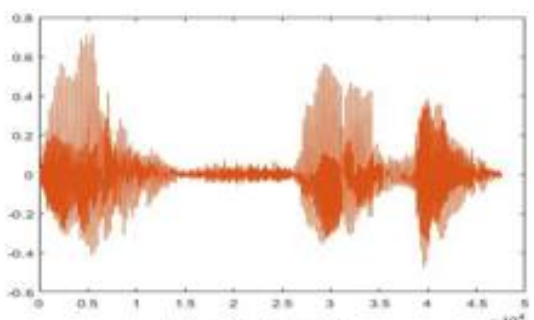

الطربقة التثاخل

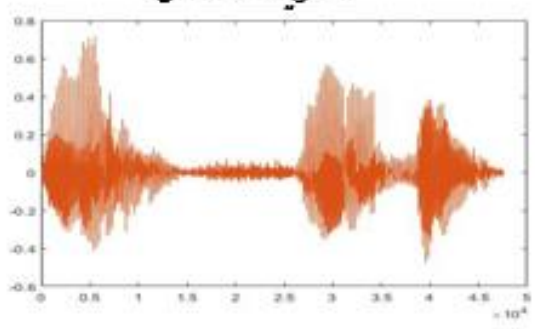

الطربقة التثاخل مع التنعيّم

الثكل (4) يمثل نطق كلمة "أحمَد" بالطرق الاربعة.

ومن هذه الاشكال نستتتج البيانات التالية:

الجدول (1) البيانات الناتجة من الموجة الصوتية التي تمثل نطق كلمة "أحمَد" بالطرق الاربعة

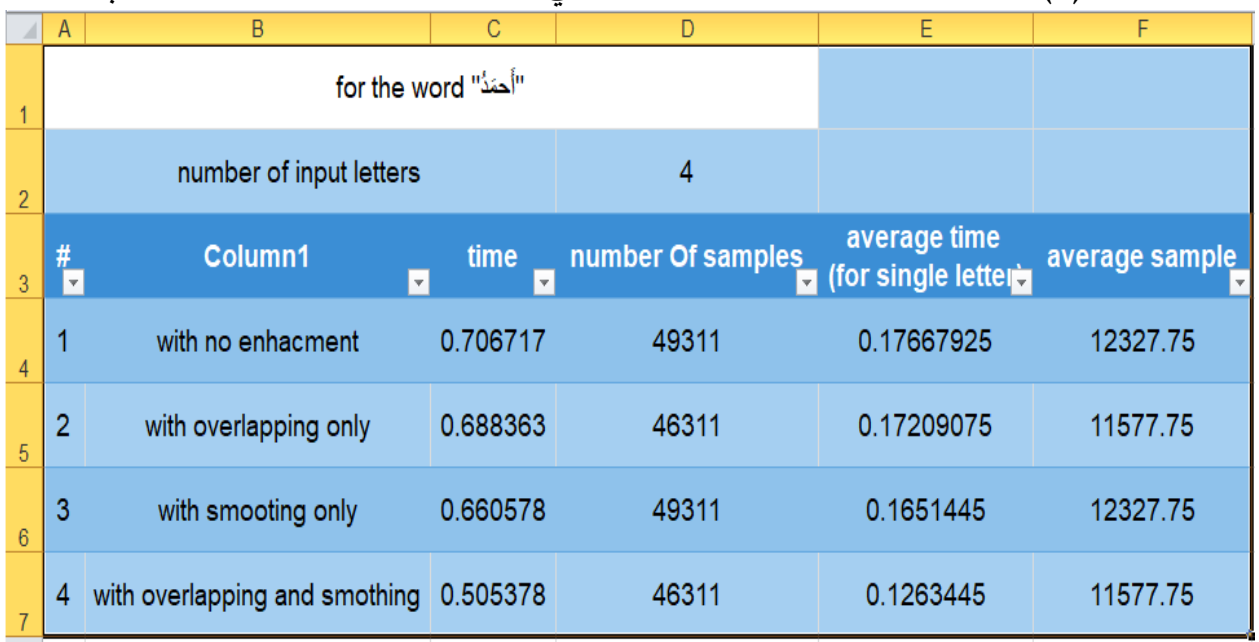


تم اعتمـاد دالــة حسـاب وقـت التنفيـذ (time) مـن بدايـة التقطيع والتحويـل الـى نهايـة عمليـة نطق الكـلام والموجودة في برنامج الماتلاب وهي دالة tic toc للحصول على أقصر وقت للتنفيذ. 8. الرسوم البياني للردود على نماذج الاستبيان المرسل عبر شبكة الانترنت الزسبوم النبياني للزدود على نماذج الاستبيان الدرسل عبر شبكة الانترنت

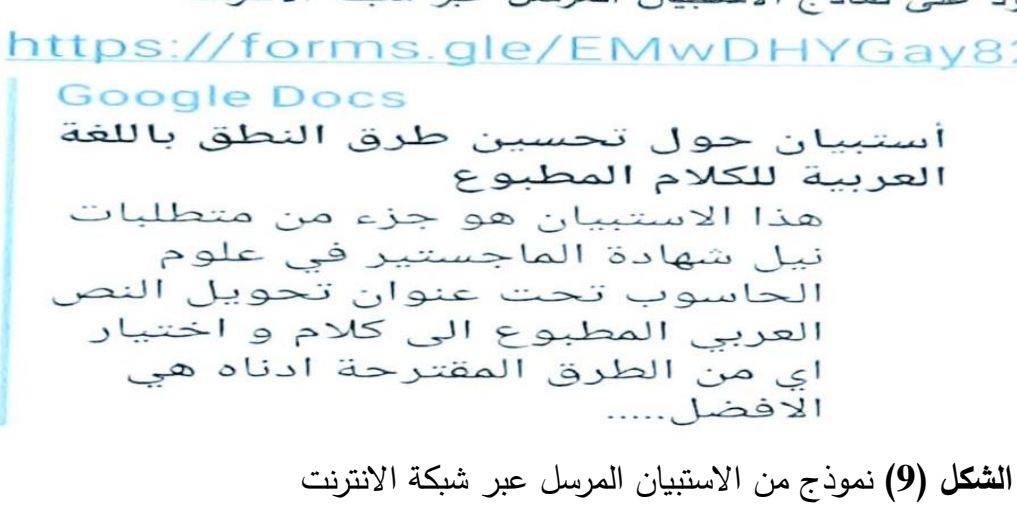

عنوان السؤال: هل أنت مختص بدراسة اللغة العربية؟ عدد الردود: 159 ردًا من خلال خمسة ايام فقط 1.9 عدد الاشخاص المختصين باللغة العربية: حيث كانت العينة مكونة من 23 شخص.

\begin{tabular}{|c|c|c|c|c|}
\hline \multirow{2}{*}{ الامج بين التداخل والتنعيم } & \multirow{2}{*}{ 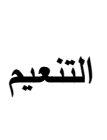 } & \multirow{2}{*}{ التداخل } & \multirow{2}{*}{ التجميع } & الطريقة \\
\hline & & & & 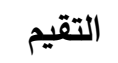 \\
\hline 5 & 4 & 4 & 6 & مقبول \\
\hline 6 & 5 & 10 & 8 & جيد \\
\hline 12 & 14 & 9 & 9 & جيد جدا \\
\hline
\end{tabular}

2.9 عدد الاشخاص المختصين وغير المختصين باللغة العربية: حيث كانت العينة مكونة من 157 شخص. حيث تم الاستفادة من هذا العدد عن طريق سماع الطرق الأربعة.

\begin{tabular}{|c|c|c|c|c|}
\hline \multirow{2}{*}{ الامجج بين التداخل والتتعيم } & \multirow{2}{*}{ التنعيم } & \multirow{2}{*}{ التداخل } & \multirow{2}{*}{ التجميع } & الطريقة \\
\hline & & & & التقيم \\
\hline 60 & 74 & 79 & 99 & مقبول \\
\hline 53 & 59 & 61 & 37 & جيد \\
\hline 46 & 25 & 18 & 22 & جيد جدا \\
\hline
\end{tabular}

نستنتج من الجدولين السابقين ان طريقة الدمج بين التداخل والتنعيم هي الأفضل من ناحية وقت التنفيذ ومن ناحية التقطيع 
في هذا البحث من ناحية تقييم الأداء، تم استخدام أربع طرق للمقارنة والحصول على أفضل نتائج للنطق،

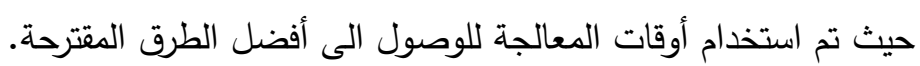
بعد تطبيق الجانب العملي من دراسة حول تحويل النص العربي السى كـلام منطوق والحصول على النتائج وتقييمها ومناقشتها، تم التوصل إلى الاستتتاجات الآتية: تم الحصول على نتائج من الكلام بعد تحقيق شَرطيّ الوضوح والفهم بنسبة عالية لهذه المرحلة وتم تقليل حجم

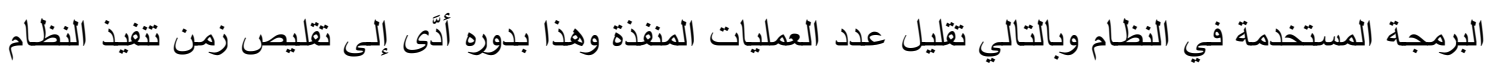

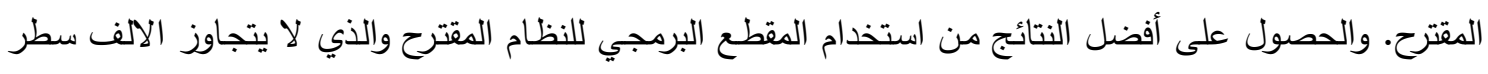

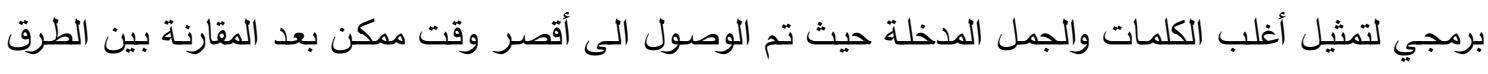

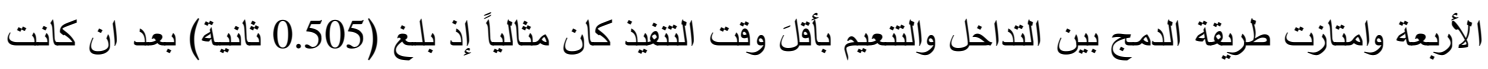

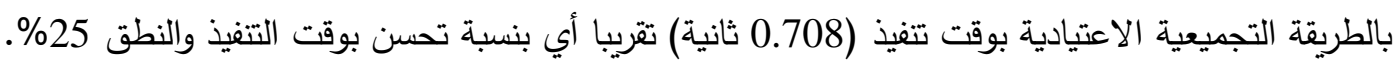




$$
\begin{aligned}
& \text { المصادر } \\
& \text { أنيس ابراهيم (1965) في اللهجات العربية ط3 مكتبة الأنجلو مصرية القاهرة ص:33 } \\
& \text { الصنغير محمود (2018) المباحث التشكيلية لأصوات اللغـة العربية في كتاب الخصـائص لابن جنى، } \\
& \text { المجلة الجامعة العدد20، المجلد الثاني- أكتوبر -2018م. } \\
& \text { ابن جني، أبو الفتح عثمان بن جني الموصلي (392ه) الخصائص الهيئة المصرية العامة للكتاب ط.4 } \\
& \text { الكسائي علي بن حمزة بن عبد الله الأسدي (189هـ) مشتبهات القرآن تحقيق محمد محمد داود، دار }
\end{aligned}
$$

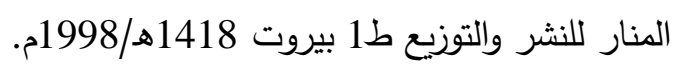

$$
\begin{aligned}
& \text { ص44-46 }
\end{aligned}
$$

لمعرفة بعض هذه البرامج الحاسوبية ينظر : المصدر السابق، ص46-47، د.ناصر عبد الرزاق الموافي،

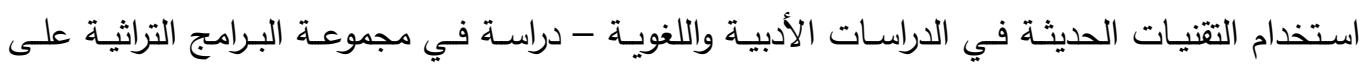
الحاسوب، ملخص بحث مقدم للمؤتمر العلمي الأول لقم اللغة العربية وآدابها بالجامعة الأردنية (آفاق

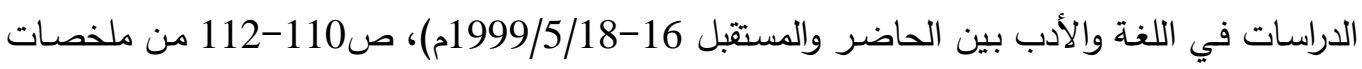
أبحاث هذا المؤتمر، لغويات الكمبيوتر العربية من الخيال إلى الواقع، مجلة بايت الثرق الأوسط كانون

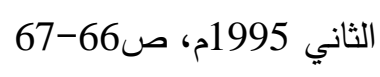

منصور بن محمد الغامدي، حسني المحتسب ومصطفى الثافعي (2004): قوانين الفونولوجيا العربية،

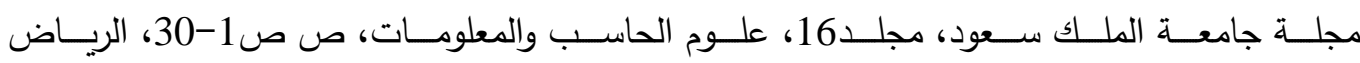

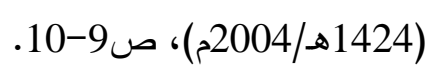

[9] Guellil I., Saâdane H., Azouaou F. Gueni B., Nouvel D (2019): Arabic natural language processing: An overview, Journal of King Saud University-Computer and Information Sciences

[10] Sanchis, R., Andrés, B., \& Poler, R. (2018). Text-To-Speech Applications to Develop Educational Materials. INTED proceedings (Online), 6085-6093.

[11] Herawati, Y.W. (2016). Integrating character education and local genius through balabolka in teaching listening. LiNGUA, 11(1), 21. 
[12] Zerrouki, T., \& Balla, A. (2017). Tashkeela: Novel corpus of Arabic vocalized texts, data for auto-diacritization systems. Data in brief, 11, 147.

[13] Kayte, M.S.N. (2018). Text-To-Speech Synthesis System for Marathi Language Using Concatenation Technique. Doctorate Thesis, Babasaheb Ambedkar Marathwada University, India.

[14] Fahmy, F.K., Khalil, M.I., \& Abbas, H.M. (2020, September). A Transfer Learning End-to-End Arabic Text-To-Speech (TTS) Deep Architecture. In IAPR Workshop on Artificial Neural Networks in Pattern Recognition (pp. 266-277). Springer, Cham.

[15] Zangar, I., Mnasri, Z., Colotte, V., Jouvet, D., \& Houidhek, A. (2018, June): Duration modeling using DNN for Arabic speech synthesis.

[16] terpconnect.umd.edu/ toh/spectrum/Smoothing.html 